\title{
Rituximab Twice Weekly for Refractory Thrombotic Thrombocytopenic Purpura in a Critically III Patient with Acute Respiratory Distress Syndrome
}

\author{
Bahjat Azrieh $^{a} \quad$ Arwa Alsaud $^{a} \quad$ Khaldun Obeidat $^{a} \quad$ Amr Ashour $^{a}$ \\ Seham Elebbi $^{a} \quad$ Shehab F. Mohamed $^{\mathrm{b}}$ Mohamed Adel Abdelaty $^{\mathrm{b}}$ \\ Abdelraouf Akkarib Abdurrahman Ali Elbuzidi ${ }^{b}$ Mohamed A. Yassin ${ }^{b}$ \\ aDepartment of Medical Education, Hamad Medical Corporation, Doha, Qatar; \\ ${ }^{b}$ National Center for Cancer Care and Research, Hamad Medical Corporation, Doha, Qatar
}

\author{
Keywords \\ Thrombotic thrombocytopenic purpura - Thrombocytopenia · Platelet disorder · Refractory \\ disease $\cdot$ Relapse $\cdot$ Rituximab
}

\section{Abstract}

Thrombotic thrombocytopenic purpura (TTP) is a rare, serious, life-threatening disease characterized by microangiopathic hemolytic anemia, thrombocytopenia, and hypercoagulability. The etiology is a deficiency of ADAMTS13 which is usually caused by acquired antibodies. Plasma exchange and steroids is the standard of care in the treatment of TTP. However, there are refractory cases of TTP which require further management. Rituximab appears to be a safe and effective therapy for refractory and relapsing TTP. Here we report a challenging case of TTP that responded to treatment with rituximab twice weekly. According to our knowledge, rituximab twice weekly has never been used for TTP before.

\section{Introduction}

Thrombotic thrombocytopenic purpura (TTP) is a rare, serious, life-threatening disease characterized by microangiopathic hemolytic anemia, thrombocytopenia, and hypercoagulability. The classic pentad of thrombocytopenia, hemolytic anemia, fever, acute kidney injury, 
Azrieh et al.: Rituximab Twice Weekly for Refractory Thrombotic Thrombocytopenic

and altered mental status, occurs in a small fraction of patients. However, the diagnosis is based on the presence of thrombocytopenia and microangiopathic hemolytic anemia.

The etiology is a deficiency of ADAMTS13. ADAMTS13 (A Disintegrin-like and Metalloproteinase with a Thrombospondin type 1 motif, member 13) - also known as von Willebrand factor-cleaving protease - is a zinc-containing metalloprotease enzyme that cleaves von Willebrand factor (vWF), a large protein involved in blood clotting. It is secreted into the blood and degrades large vWF multimers, decreasing their activity [1].

When these molecules are not properly cleaved, unprompted platelet aggregation ensues, activating the coagulation cascade and resulting in platelet consumption. vWF molecules cause the shearing of red blood cells, evoking thrombocytopenia and hemolytic anemia. The clots formed by this process can cause emboli, which can block blood vessels and lead to endorgan damage. Deficiency of ADAMTS13 is usually caused by an acquired antibody. In very rare cases, an ADAMTS13 deficiency is inherited in an autosomal recessive pattern, such as in Upshaw-Schulman syndrome [2].

Plasma exchange and steroids is the standard of care in the treatment of TTP. However, there are refractory cases of TTP which require further management. Rituximab is used as salvage treatment for refractory TTP. Rituximab is a chimeric (human-mouse) monoclonal antibody against the protein CD20, which is primarily found on the surface of lymphocytic B cells [3]. When it binds to this protein it triggers cell death [4]. B cells are responsible for antibody production. Thus, killing B cells will eliminate the source of antibodies against ADAMTS13. Rituximab is used to treat certain autoimmune diseases and types of cancer [4]. Here we report a challenging case of refractory TTP that responded to treatment with rituximab twice weekly.

\section{Case Presentation}

A 32-year-old, male patient, with a known case of ulcerative colitis for 12 years prior to presentation on mesalamine, presented with watery, non-bloody diarrhea, occurring 10-12 times per day for 4 days prior to presentation. He noted yellow discoloration of his eyes (jaundice) and also reported pain in both knees. No other symptoms were present on admission. He was treated initially supportively with IV fluids.

Laboratory studies showed anemia, thrombocytopenia, mixed hyperbilirubinemia, and high creatinine. Peripheral blood smear showed evidence of microangiopathic hemolytic anemia. ADAMTS13 level was less than 5\%. The diagnosis of TTP was made.

A central line was inserted, and daily plasma exchange was started along with oral prednisolone $40 \mathrm{mg}$ daily, then increased to $60 \mathrm{mg}$ daily. Initial improvement occurred. Platelet count rose from 10,000 to 129,000. Diarrhea improved after giving steroids.

A few days later, platelet count dropped again, and the patient had multiple episodes of generalized tonic-clonic seizures. CT scan and MRI of the head were normal. He was started on levetiracetam prophylaxis for seizures. The decision was made to start rituximab once weekly. He received 2 doses of rituximab.

After that, he had fever, dyspnea, desaturation, and decreased level of consciousness. He was diagnosed with aspiration pneumonia. He required endotracheal intubation and mechanical ventilation. He was transferred to the intensive care unit (ICU) and started on antibiotics empirically.

In the ICU, the patient had bleeding from the endotracheal tube and developed acute respiratory distress syndrome (ARDS), most likely secondary to pulmonary hemorrhage. He also had bleeding per rectum for 1 day with a drop in hemoglobin that required transfusion of 5 units of packed red blood cells. He was intubated for 1 month and our impression was 
Azrieh et al.: Rituximab Twice Weekly for Refractory Thrombotic Thrombocytopenic Purpura in a Critically III Patient with Acute Respiratory Distress Syndrome

that the patient would not be able to recover, so rituximab was started twice weekly instead of once weekly along with daily plasma exchange and steroids. After that, the patient's respiratory status improved, and he was extubated. Five days after extubation, platelet count rose to normal level, creatinine dropped to a normal level, and the patient improved clinically. He received a total of 49 sessions of plasma exchange and 7 doses of rituximab.

\section{Discussion}

Similar to other autoimmune conditions, treatment is based on removing circulating antibodies and restoring proper levels of ADAMTS13. This is accomplished by plasma exchange as well as intravenous steroids. Although patients may present with severe anemia and thrombocytopenia, the transfusion of red blood cells and platelets is contraindicated before treating the underlying etiology because the circulating antibodies can attack the transfused blood products causing worsening of the condition. Refractory cases that do not respond to plasma exchange or steroids can benefit from rituximab. Relapsing and refractory cases have also been treated with immunosuppressive therapies such as vincristine, cyclophosphamide, cyclosporine A, or even splenectomy. Response to treatment is measured mainly by a symptomatic response as well as levels of lactate dehydrogenase (LDH), schistocytes on blood smears, and platelets. Levels of ADAMTS13 are usually measured at follow-up. Patients who receive prompt treatment with plasma exchange have a very favorable prognosis, with an average survival rate of 90\% [5].

A recent expert statement on the ICU management of patients with TTP suggested the following: The lack of platelet normalization by day 5 associated with persistent signs of hemolysis (high LDH level) and/or severe cardiac or neurological manifestations defines unresponsive or refractory TTP [6]. Older age, cerebral involvement, and persistently high LDH may help identify TTP patients at increased risk of early death [6]. All patients diagnosed with TTP must be initially admitted to an ICU to monitor and manage organ dysfunctions and to provide urgent plasma exchange therapy [6]. Experts suggest that ICU discharge be allowed when plasma exchange can be tapered or stopped, when signs of hemolysis disappear, and platelet count increases over $150,000 / \mathrm{mL}$, which usually correlates with cardiac and neurological improvements [6]. Experts suggest that rituximab should be used in patients with relapsing autoimmune TTP and should also probably be used as a first-line therapy in severe TTP [6]. Our patient had an improvement initially and then deteriorated. The use of rituximab in our case boosted steroids and plasma exchange in achieving remission.

A retrospective cohort study published in the journal Clinical Medicine Insights: Blood Disorders in 2015 concluded that rituximab was a safe and effective therapy for refractory and relapsing TTP. Researchers reviewed the efficacy and safety of rituximab in 9 cases of refractory TTP and 1 case of relapsing TTP. Complete remission was achieved in all cases. Rituximab was well tolerated in all cases, and no major side effects were seen except for mild allergic reaction (such as fever, rigors, itching) mainly after the first dose [7].

Whether rituximab should be a frontline treatment for autoimmune TTP or only reserved for patients with a suboptimal response to initial treatments remains controversial. A recent systematic review and meta-analysis compared rituximab with conventional therapy and showed that patients receiving rituximab to induce disease remission had a significantly lower relapse rate than those given conventional treatment. Furthermore, the conventional treatment group had a significantly higher mortality rate than the rituximab group during the follow-up. Rituximab offered high efficacy for the prevention of relapses and lower mortality rate in cases of acquired TTP [8]. 
According to our knowledge, rituximab twice weekly has never been used before. Our patient was refectory to plasma exchange although it was done daily for more than 2-3 weeks along with treatment with steroids. We used rituximab weekly, but the patient did not show any improvement, so we increased it to twice per week trying to save his life and we succeeded.

A review published in the American Journal of the Medical Sciences in 2001 concluded that ARDS can be a manifestation of TTP. The authors recommended that all patients with ARDS and unexplained thrombocytopenia should be evaluated for unrecognized TTP [9].

A case report published in 1998 also indicated that ARDS can be a manifestation of TTP. The patient was treated with plasma exchange till he recovered from both ARDS and TTP [10].

Another study published in the Journal of Investigative Medicine in 2002 reviewed the clinical manifestations of TTP in 74 patients and found that ARDS was diagnosed in 13 patients. A poor prognosis was evident when ARDS was present, with an overall survival rate of $46 \%$ [11].

ARDS, gastrointestinal hemorrhage, and mesenteric ischemia were all reported as possible complications of TTP. Our patient had ARDS and gastrointestinal hemorrhage.

\section{Conclusion}

TTP is a serious hematological disorder with a considerable number of refractory and relapsing cases. Rituximab addition to plasma exchange and steroids is a good strategy. Rituximab could be lifesaving for many of these patients. Experts recommend the use of rituximab, but there is still no clear guideline on this use.

Rituximab may even replace the conventional therapy in the future based on many positive outcomes reported in studies and in clinical practice. Based on our experience with the case reported here, we suggest that rituximab can be used twice weekly instead of once weekly for refractory TTP. However, further studies are required to prove this.

\section{Acknowledgement}

We thank Qatar National Library for funding this article and we thank the internal medicine residency program research group at Hamad Medical Corporation.

\section{Statement of Ethics}

This article was approved by Hamad Medical Corporation Medical Research Center. This research was conducted ethically in accordance with the World Medical Association Declaration of Helsinki. The subject in this paper has given their informed consent to publish their case.

\section{Disclosure Statement}

The authors have nothing to disclose.

\section{Funding Sources}

This article was funded by Qatar National Library. 
Azrieh et al.: Rituximab Twice Weekly for Refractory Thrombotic Thrombocytopenic Purpura in a Critically III Patient with Acute Respiratory Distress Syndrome

\section{Authors Contributions}

Bahjat Azrieh: Writing the manuscript; Arwa Alsaud: Clinical management; Amr Ashour: Clinical management; Khaldun Obeidat: Clinical management; Seham Elebbi: Clinical management; Shehab F. Mohamed: Clinical management; Mohamed Adel Abdelaty: Clinical management; Abdelraouf Akkari: Clinical management; Abdurrahman Ali Elbuzidi: Clinical management; Mohamed A. Yassin: Writing and editing.

\section{References}

1 Levy GG, Motto DG, Ginsburg D. ADAMTS13 turns 3. Blood. 2005 Jul;106(1):11-7.

2 National Heart, Lung, and Blood Institute (NHLBI). Thrombotic thrombocytopenic purpura. 2019. https:// www.nhlbi.nih.gov/health-topics/thrombotic-thrombocytopenic-purpura.

3 Bosch X, Ramos-Casals M, Khamashta MA, editors. Drugs targeting B-cells in autoimmune diseases. Springer Science \& Business Media; 2013. pp. 1-4. ISBN 978-3-0348-0706-7.

4 "Rituximab". The American Society of Health-System Pharmacists. Archived from the original on 27 March 2016. Retrieved 8 December 2016. https://www.drugs.com/monograph/rituximab.html.

5 Blombery P, Scully M. Management of thrombotic thrombocytopenic purpura: current perspectives. J Blood Med. 2014;5:15-23.

6 Azoulay E, Bauer PR, Mariotte E, Russell L, Knoebl P, Martin-Loeches I, et al. Nine-i Investigators. Expert statement on the ICU management of patients with thrombotic thrombocytopenic purpura. Intensive Care Med. 2019 Nov;45(11):1518-1539.

7 Omri HE, Taha RY, Gamil A, Ibrahim F, Sabah HA, Mahmoud ZO, et al. Efficacy and safety of rituximab for refractory and relapsing thrombotic thrombocytopenic purpura: a cohort of 10 cases. Clin Med Insights Blood Disord. 2015 May;8:1-7.

8 Owattanapanich W, Wongprasert C, Rotchanapanya W, Owattanapanich N, Ruchutrakool T. Comparison of the long-term remission of rituximab and conventional treatment for acquired thrombotic thrombocytopenic purpura:a systematic review and meta-analysis. Clin Appl Thromb Hemost. 2019 Jan-Dec;25: 107602961882530.

9 Chang JC, Aly ES. Review. Acute respiratory distress syndrome as a major clinical manifestation of thrombotic thrombocytopenic purpura. Am J Med Sci. 2001 Feb;321(2):124-8.

10 Chang JC, Gupta S. Acute respiratory distress syndrome and non-occlusive mesenteric ischemia as major clinical manifestations of thrombotic thrombocytopenic purpura: complete remission following exchange plasmapheresis. J Clin Apher. 1998;13(4):190-2.

11 Chang JC, Kathula SK. Various clinical manifestations in patients with thrombotic microangiopathy. J Investig Med. 2002 May;50(3):201-6. 